Educación Física y Ciencia, vol. 23, n³, e190, julio-septiembre 2021. ISSN 2314-2561

Universidad Nacional de La Plata.

Facultad de Humanidades y Ciencias de la Educación.

Departamento de Educación Física

\title{
Exercícios Físicos para Mulheres com Varizes: Treinar condicionamento geral ou membros inferiores?
}

\author{
Ícaro do Carmo Carvalho \\ Universidade Federal de Pernambuco - UFPE, \\ Programa de Pós-graduação em Educação Física, Brasil \\ icaroicc@hotmail.com \\ iD https://orcid.org/0000-0002-5955-0176 \\ Daniela Karina da Silva Ferreira \\ Universidade Federal de Pernambuco - UFPE, \\ Programa de Pós-graduação em Educação Física, Brasil \\ danielakarina@hotmail.com \\ (DD https://orcid.org/0000-0003-1786-7965
}

Physical Exercises for Women with Varicose Veins: Training general conditioning or lower limbs?

Ejercicios Físicos para Mujeres con Varices: ¿Entrenar del acondicionamiento general o de las extremidades inferiores?

\begin{abstract}
Resumo:
Esse estudo comparou o efeito de dois programas de treinamento sobre a aptidão física e qualidade de vida em mulheres com Varizes, sendo um programa de condicionamento físico geral (CFG) e o outro de fortalecimento de membros inferiores (MMII) aplicado a 2 grupos de voluntárias, praticantes regulares de exercício físico, por 12 semanas. Foram coletados, pré e pós treinamento, dados de qualidade de vida, perimetria de panturrilha, goniometria do tornozelo, teste de ponta de pé e degrau. Nove mulheres do programa CFG ( $54 \pm 18$ anos) e onze do programa MMII ( $58 \pm 8$ anos) concluíram a intervenção. Comparando a magnitude de variação nos resultados de cada teste e entre os programas por teste $t$ independente, as participantes do programa MMII apresentaram melhor desempenho nos testes de aptidão física e qualidade de vida que as do programa CFG, com diferença significativa nos resultados de resistência muscular $(\mathrm{p}<0,01$ para repetições e $\mathrm{p}=0,02$ para tempo de execução no teste de ponta de pé) e flexibilidade $(\mathrm{p}<0,01)$. Concluiu-se que um programa específico de fortalecimento de membros inferiores possibilita melhores resultados em aptidão física em relação a um programa de condicionamento geral.
\end{abstract}

PalaVras-ChaVe: Varizes, Aptidão Física, Força Muscular.

\section{Abstract:}

This study compared the effect of two training programs on physical fitness and quality of life in women with varicose veins, one being a general fitness program (CFG) and the other for strengthening lower limbs (MMII by its acronym in Portuguese) applied to 2 groups of volunteers, regular practitioners of physical exercise, for a period of 12 weeks. Data about quality of life, calf perimeter, ankle goniometry, heel rise test and step test were collected, pre and post training. Nine women from the CFG program ( $54 \pm$ 18 years) and eleven from the MMII program ( $58 \pm 8$ years) completed the intervention. Comparing the magnitude of variation in the results of each test and between the programs by independent $t$-test, the participants in the MMII program showed better performance in the tests of physical fitness and quality of life than those of the CFG program, with a significant difference in the results of muscular endurance $(\mathrm{p}<0.01$ for repetitions and $\mathrm{p}=0.02$ for execution time in the toe test) and flexibility $(\mathrm{p}<0.01)$. It was concluded that a specific program to strengthen lower limbs allows better results in physical fitness compared to a general conditioning program.

KEYWORDS: Varicose veins, Physical fitness, Muscle strength.

\section{Resumen:}

Este estudio comparó el efecto de dos programas de entrenamiento sobre la aptitud física y la calidad de vida en mujeres con varices, un programa de acondicionamiento físico general (CFG) y otro de fortalecimiento de miembros inferiores (MMII) aplicado a 2 grupos de voluntarios, practicantes regulares de ejercicio físico, durante 12 semanas. Se recopilaron datos previos y posteriores al entrenamiento sobre la calidad de vida, la perimetría de la pantorrilla, la goniometría del tobillo, las pruebas de pasos y de puntillas.

\section{Recepción: 28 de noviembre de 2020 | Aprobación: 12 de junio de 2021 | Publicación: 01 de julio de 2021}


Nueve mujeres del programa CFG (54 \pm 18 años) y once del programa MMII (58 \pm 8 años) completaron la intervención. Al comparar la magnitud de la variación en los resultados de cada prueba y entre programas mediante una prueba $t$ independiente, las participantes del programa MMII obtuvieron mejores resultados en las pruebas de aptitud física y calidad de vida que las del programa CFG, con una diferencia significativa en los resultados de resistencia muscular. $(\mathrm{p}<0.01$ para repeticiones y $\mathrm{p}=$ 0.02 para tiempo de ejecución en la prueba de puntillas) y flexibilidad $(\mathrm{p}<0.01)$. Se concluyó que un programa específico para el fortalecimiento de las extremidades inferiores permite mejores resultados en la aptitud física en comparación con un programa de acondicionamiento general.

Palabras clave: Varices, Aptitud Física, Fuerza Muscular.

\section{INTRODUÇÃo}

Os sintomas da Doença Venosa Crônica debilitam os níveis de força, resistência e mobilidade dos membros inferiores interferindo na capacidade de desempenhar atividades físicas cotidianas. Além disso, o aspecto estético das varizes impacta na qualidade de vida, sendo causa de isolamento social, absenteísmo e, em casos mais graves, aposentadoria por invalidez (Lima et al., 2002). A alta prevalência, cronicidade e morbidade tornam a DVC um problema de Saúde Pública (Flávia de Jesus Leal et al., 2016; R. M. F. Moura, Gonçalves, Navarro, Britto, \& Dias, 2008) aumentando a necessidade de assistência em saúde, tanto na atenção básica como na alta complexidade.

O exercício físico tem sido apontando como uma estratégia de baixo custo na prevenção, controle e tratamento da DVC. Há estudos envolvendo programas de exercícios avaliando o efeito do fortalecimento da musculatura da panturrilha sobre diversos aspectos da DVC como hemodinâmica venosa, sintomas e qualidade de vida (Alberti, Petroianu, Corrêa, \& Silva, 2008; Davies, Bull, Farrelly, \& Wakelin, 2007; Kan \& Delis, 2001; Lima et al., 2002). Considerando que a bomba muscular da panturrilha tem papel importante em relação ao fluxo sanguíneo e se relaciona a DVC (Youn \& Lee, 2019), em boa parte desses estudos, enfatizase a utilização de exercícios específicos para fortalecimento e mobilidade da região da panturrilha e tornozelo, combinados ou não com exercícios aeróbicos (Ercan, Çetin, Yavuz, Demir, \& Atalay, 2017; Szewczyk et al., 2010). São poucas as propostas de treinamento mais complexas envolvendo outros grupos musculares, além da musculatura da panturrilha, impossibilitando uma comparação de seus possíveis efeitos.

Assim, este estudo teve como objetivo comparar a magnitude de variação nas variáveis de aptidão física e qualidade de vida em dois programas de treinamento, sendo um com ênfase na musculatura de membros inferiores e outro de condicionamento físico geral.

\section{MÉtodos}

\section{Participantes}

Foram recrutadas mulheres com DVC praticantes regulares de atividade física, há pelo menos três meses, no Programa Academia da Cidade (Recife/PE) e no projeto Vida Ativa da Universidade Federal de Pernambuco (UFPE). Sendo excluídas as que fizeram algum tratamento para DVC, apresentassem úlceras ativas, doença ortopédica, neurológica ou sistêmica que afete a bomba muscular da panturrilha ou dificulte a prática de exercícios físicos e aquelas com diabetes ou hipertensão descompensados. As voluntárias foram submetidas à uma avaliação inicial, intervenção de 12 semanas de atividades físicas sob um dos dois programas de treinamento desenvolvidos para este estudo, definido previamente por sorteio, e uma avaliação final. O estudo foi aprovado pelo comitê de ética em pesquisa do Centro de Ciências da Saúde da Universidade Federal de Pernambuco (parecer $n^{\circ}$ 3373609), bem como foram obtidos os termos de anuência junto a Secretaria de Saúde do Município do Recife e do Laboratório de Cinesiologia e Avaliação Funcional da UFPE (LACAF). 


\section{Procedimentos}

As participantes recrutadas, no período de junho a julho de 2019, atendendo aos critérios de inclusão e exclusão, e após assinatura do TCLE, foram submetidas a aplicação de questionário sociodemográfico e a uma avaliação clínica da DVC, por uma fisioterapeuta, utilizando a classificação CEAP (Rabe \& Pannier, 2012).

Como as intervenções foram realizadas nos respectivos locais de atividade das participantes, os programas a serem seguidos foram definidos por sorteio de acordo com as turmas e os polos de origem das voluntárias, de forma que quatro turmas foram sorteadas para seguir o programa de Condicionamento Físico Geral (programa CFG), e três turmas seguiram o programa de exercícios específico para Membros Inferiores (programa MMII).

Os dois grupos participaram das atividades 4 vezes por semana, durante o período de 12 semanas (entre agosto e novembro de 2019), perfazendo até 48 sessões de treino. Foi feito controle de frequência, através de lista de presença, para verificar participação mínima de 70\% nas atividades. Foram realizadas ao início e ao final das 12 semanas de intervenção a aplicação do questionário de qualidade de vida, as medidas e testes para avaliação das variáveis de aptidão física, coletados por avaliadores previamente treinados, cegos para os grupos e todas as informações coletadas armazenadas eletronicamente, descritas na respectiva ordem:

\section{Qualidade de Vida}

Com utilização do instrumento VEINES QoL/Sym (versão traduzida e adaptada transculturalmente), composto por 26 itens distribuídos em 8 questões, avaliou-se a presença e intensidade de sintomas da DVC bem como o impacto destes no comportamento e nas atividades de vida diária, laborais e sociais. O questionário produz dois escores, um relacionado ao impacto da DVC na QV (VEINES QoL) e outro relacionado aos sintomas (VEINES Sym), que são obtidos através da padronização de cada item em escore $\mathrm{Z}$ e cálculo conforme protocolo original em que os maiores valores do escore final indicam melhor resultado (R. M. F. de; Moura, Gonçalves, Navarro, Britto, \& Dias, 2011).

\section{IMC e Perimetria de Panturrilha}

Para aferição do IMC, as medidas de peso corporal e estatura foram coletadas em balança digital (graduada em $100 \mathrm{~g}$ ) com estadiômetro (graduado em $\mathrm{cm}$ ). Com uso de fita métrica inelástica foi coletada a circunferência da panturrilha (registrada em $\mathrm{cm}$ ), com a voluntária sentada e pés apoiados no chão (C. Belczak et al., 2004).

\section{Flexibilidade da articulação do tornozelo}

Realizado por Goniometria do tornozelo, com a participante deitada em decúbito dorsal e pés fora da mesa de avaliação, e pés em posição neutra $\left(0^{\circ}\right)$, foi solicitado que ela realize um movimento máximo de dorsiflexão, anotando a angulação com um goniômetro universal, seguido de um movimento máximo de flexão plantar, também registrando a medida em graus $\left(^{\circ}\right)$. Procedimento realizado em ambos os pés (Flávia de Jesus; Leal, Couto, Silva, \& Tenório, 2015).

\section{Resistência muscular da Panturrilha}

Para avaliar a função da bomba muscular da panturrilha foi proposto o Teste de Ponta de Pé (TPP), no qual a avaliada, em pé, com uma das mãos apoiada na parede, realiza um movimento de flexão plantar 
bilateral até a máxima altura possível. Com esta altura demarcada servindo de referência ao avaliador, ao comando inicial, realizar o máximo de flexões possível, atingindo o aparato demarcador da altura máxima, até que não mais alcance a altura de referência por duas vezes consecutivas ou informe exaustão. Foi registrado o número de repetições, o tempo de execução, bem como calculada a velocidade de execução (Monteiro et al., 2017; Pereira, Oliveira, Cruz, Souza, \& Cunha Filho, 2008).

\section{Resistência aeróbica e Trabalho Muscular}

Avaliados por meio do Teste do Degrau de 4 minutos (TD4), no qual a avaliada em pé, de frente a um step de $20 \mathrm{~cm}$ de altura, ao comando inicial, realiza movimentos de subir e descer o degrau o maior número de vezes possível durante 4 minutos. $\mathrm{O}$ avaliador, fornecendo estímulo verbal à voluntária, contava o número total de subidas realizadas. A avaliada poderia pausar a execução, e retornar assim que sentir-se bem para fazê-lo, sem que o cronômetro fosse parado nessa situação (Marrara, Marino, \& Jamami, 2012; fórmula Murayama et al., 2014).

A de cálculo do trabalho da força-peso (força multiplicada pela distância de deslocamento da carga) foi aplicada ao teste de modo que o peso (em $\mathrm{Kg})$ da avaliada foi multiplicado pela altura do step $(0,2 \mathrm{~m})$ e pelo número de subidas.

\section{Programas de Intervenção}

As sessões de treino duraram aproximadamente 45 minutos, seguindo o padrão de 10 minutos de alongamento e aquecimento, 30 minutos da parte principal, e 5 minutos de resfriamento. A intensidade e carga das atividades foi mantida durante todo o período de intervenção, com exercícios executados com peso corporal ou com halteres de 2 ou $3 \mathrm{~kg}$, controlados por tempo. Para padronizar as atividades, os professores de cada polo participaram de reuniões com os pesquisadores, para as instruções sobre os programas e receberam cartilha com as atividades a serem desenvolvidas. No programa CFG, foi desenvolvido um programa de Condicionamento Físico Geral, cujas atividades envolviam exercícios de resistência muscular localizada e circuito funcional envolvendo todos os grupamentos musculares, além do Step como atividade aeróbica. Enquanto no programa MMII, foi aplicado um programa específico para o treinamento dos membros inferiores, em circuito, também alternando com aulas de Step. As atividades desenvolvidas seguiram uma programação semanal alternando atividades aeróbicas com as de resistência muscular, conforme quadro 1 .

Quadro 1 - Programas de treinamento desenvolvidos para o estudo

\begin{tabular}{lcccc}
\hline Programa & Segunda & Terça & Quarta & Quinta \\
\hline CFG & RML & A eróbicos & Circuito Funcional & Aeróbicos \\
& (Abs/MMSS $)$ & STEP & & STEP \\
MMII & Aeróbicos & Circuito Funcional & Aeróbicos & Circuito \\
& STEP & (MMI) & STEP & Funcional (MMI) \\
& & & & \\
\end{tabular}




\section{RML (Abdominais/MMSS)}

As atividades de resistência muscular localizada consistiam em 10 exercícios para Membros Superiores e Abdominais, executados em sequência por 2 séries com 30 segundos de execução seguidas de 30 segundos de descanso.

\section{Circuito Funcional}

Para o grupo submetido ao programa CFG, o circuito foi composto por 6 estações, alternando exercícios aeróbicos com exercícios de força envolvendo grandes grupos musculares com halteres. A execução foi de 40 segundos, 10 segundos para troca de estação e 2 minutos de descanso ativo com caminhada ao fim de cada rotação completa no circuito. Eram executadas 2 rotações, e mais 2 com outros 6 exercícios diferentes.

Para o grupo submetido ao programa MMII, a sequência foi de 4 estações com exercícios básicos de força para membros inferiores, executados por 40 segundos, com 10 segundos para as trocas de estações e 2 minutos de descanso ao fim da rotação completa. Após três rotações, os mesmos exercícios foram realizados de forma unilateral por mais três rotações.

\section{Step}

As aulas de step seguiam o mesmo padrão tanto para as participantes de ambos os programas. Utilizando implementos emborrachados, com altura de $10 \mathrm{~cm}$, seguindo passos básicos, executados em sequência livre, não coreografados, com músicas na frequência de 128 batimentos por minuto.

\section{Aquecimento/Resfriamento}

Para ambos os programas, as aulas foram iniciadas com uma caminhada de 3 minutos, seguidas de alongamento geral, com ênfase na musculatura dos membros inferiores. Para o fechamento das aulas foram realizadas atividades de volta à calma com exercícios de mobilidade de tornozelo e pé.

\section{Análise Estatística}

Foi utilizada estatística descritiva para apresentação dos dados e variáveis em média \pm desvio padrão e teste $\mathrm{T}$ comparando os grupos pré-intervenção. A normalidade da distribuição da amostra, analisada por teste de Shapiro Wilk. Para a comparação dos dados pós-treinamento entre os dois grupos, foi calculado o delta de cada variável e comparadas as médias das diferenças através do teste $t$ independente e MannWhitney $U$ para dados não normais, adotado nível de significância de 0,05. Os dados foram analisados utilizando pacote estatístico SPSS, versão 25.0.

\section{Resultados}

Na primeira etapa da coleta de dados, das 49 voluntárias com DVC, 44 atenderam aos critérios de inclusão e exclusão. Dessas, 20 cumpriram todo o programa de treinamento com mais de $70 \%$ de frequência. A perda amostral no período de intervenção ocorreu, na maioria dos casos, por excesso de faltas às sessões de treino (figura 1). Não foi registrada intercorrência com as participantes durante a intervenção. 
Figura 1 - Fluxograma das fases do estudo, Recife-PE/Brasil, 2020.

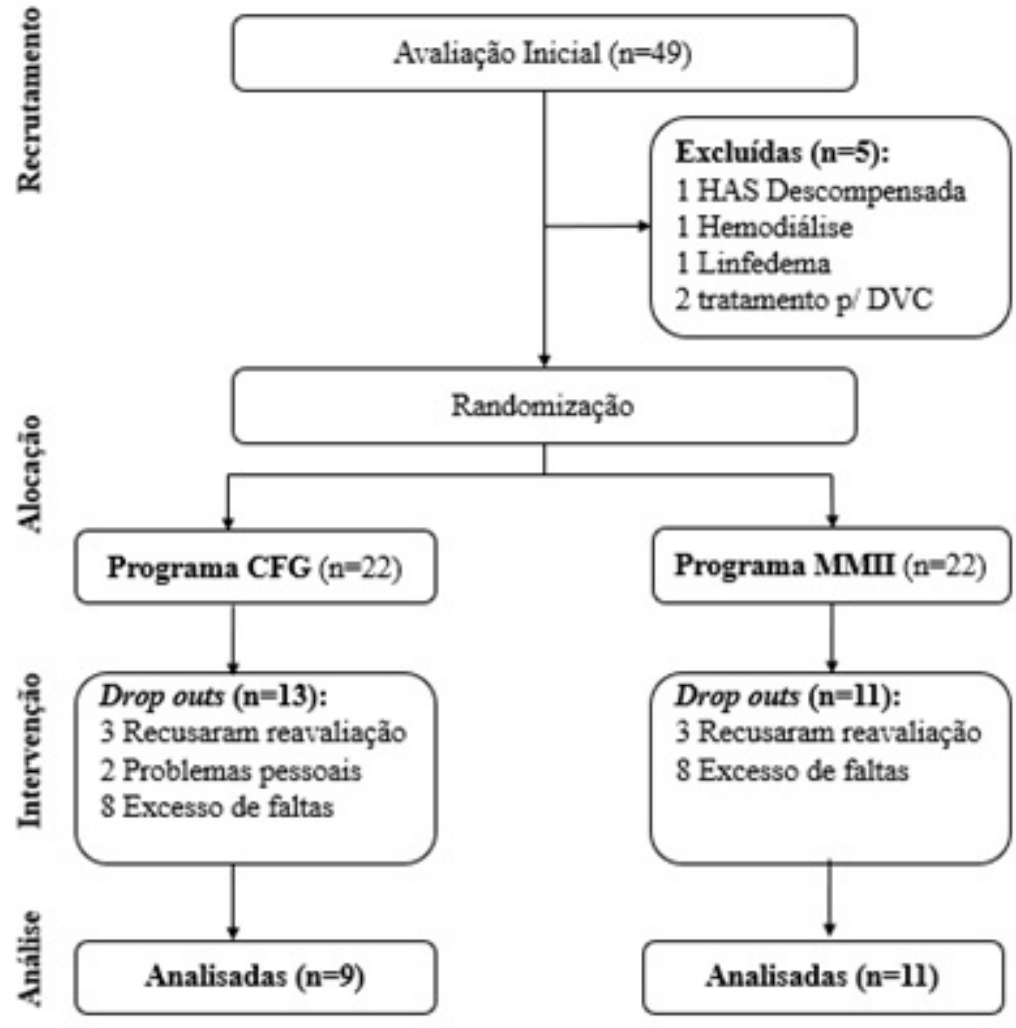

Fuente: Elaboração Própria.

A amostra participante de ambos os programas de treinamento apresentou idade média de 54 e 58 anos, respectivamente. A maior parte das voluntárias apresentou sobrepeso/obesidade (56\% no programa CFG e 91\% no programa MMII), sendo encontrada diferença significativa quanto ao IMC e flexibilidade do tornozelo entre os grupos, com valores superiores nas participantes do programa MMII. A amostra, em ambos os grupos, já participava de programa de exercícios físicos há mais de dois anos (40 meses dentre as participantes do programa CFG e 33 meses dentre as do programa MMII).

Em 77\% das participantes do programa CFG e 91\% do programa MMII há presença de varizes nos membros inferiores com classificação de severidade moderada. Em ambos os grupos, a média foi de 3 gestações por participante.

$\mathrm{Na}$ análise dos dados, comparou-se as diferenças relativas pós e pré treinamento nos resultados das medidas e testes de aptidão física obtidos pelas participantes em cada programa de treinamento, conforme apresentado na tabela 1. Desse modo, o programa MMII proporcionou melhor resultado em todas as variáveis de aptidão física, exceto em IMC. 
Tabela 1 - Comparação das medidas de Aptidão Física pré e pós treinamento nos dois programas, Recife-PE/Brasil, 2020.

\begin{tabular}{|c|c|c|c|c|c|c|c|c|c|c|c|c|}
\hline \multirow[b]{2}{*}{ Teste } & \multicolumn{5}{|c|}{ Programa CFG $(n=9)$} & \multicolumn{5}{|c|}{ Programa MMII $(\mathrm{n}=11)$} & \multirow{2}{*}{ pl } & \multirow{2}{*}{$p \Delta$} \\
\hline & Pré & DP & Pós & DP & $\Delta \%$ & Pré & DP & Pós & DP & $\Delta \%$ & & \\
\hline IMC $\left(\mathrm{kg} / \mathrm{m}^{2}\right)$ & 25,7 & 2,1 & 25,9 & 2,1 & $1 \%$ & 28,8 & 3,4 & 29,4 & 3,9 & $2 \%$ & $0,02^{*}$ & 0,20 \\
\hline Panturrilha D $(\mathrm{cm})$ & 35,8 & 1,7 & 36,2 & 2 & $1 \%$ & 36,9 & 2,5 & 38 & 3,7 & $3 \%$ & 0,31 & 0,05 \\
\hline Panturrilha E (cm) & 36,5 & 2,1 & 36,4 & 2,1 & $0 \%$ & 37,0 & 2,7 & 38,2 & 3,7 & $3 \%$ & 0,62 & 0,18 \\
\hline $\begin{array}{l}\text { Flex. Tomozelo D } \\
\left({ }^{\circ}\right)\end{array}$ & 65 & 7,5 & 64,7 & 10,1 & $-1 \%$ & 51 & 13,5 & 61,9 & 7,3 & $22 \%$ & $0,01^{*}$ & $<0,01^{*}$ \\
\hline $\begin{array}{l}\text { Flex. Tormozelo E } \\
\left({ }^{\circ}\right)\end{array}$ & 61,8 & 6,8 & 63,4 & 8,6 & $3 \%$ & 50,6 & 12,9 & 60,7 & 6,3 & $18 \%$ & $0,03^{*}$ & 0,07 \\
\hline Res. Muscular (rep) & 45 & 20 & 37 & 19 & $-19 \%$ & 31 & 16 & 53 & 19 & $68 \%$ & 0,09 & $<0,01^{*}$ \\
\hline Res. Muscular (s) & 62,0 & 33,4 & 43,8 & 14,3 & $-29 \%$ & 38,6 & 17,9 & 59,6 & 32,8 & $51 \%$ & 0,06 & $0,02 *$ \\
\hline $\begin{array}{l}\text { Res. Muscular } \\
\text { (rep/s) }\end{array}$ & 0,80 & 0,34 & 0,86 & 0,32 & $7 \%$ & 0,80 & 0,21 & 0,94 & 0,18 & $16 \%$ & 0,99 & 0,39 \\
\hline $\begin{array}{l}\text { Res. Aeróbica }\left(n^{\circ}\right. \\
\text { sub) }\end{array}$ & 105 & 25 & 121 & 23 & $15 \%$ & 88 & 19 & 116 & 28 & $33 \%$ & 0,11 & 0,10 \\
\hline $\begin{array}{l}\text { Trabalho TD4 } \\
\text { (kg.m) }\end{array}$ & 1283 & 345 & 1476 & 310 & $15 \%$ & 1129 & 474 & 1634 & 424 & $37 \%$ & 0,42 & 0,05 \\
\hline
\end{tabular}

$\mathrm{p} 1=$ teste $\mathrm{t}$ pareado entre os programas pré-treinamento; $\mathrm{p} \Delta=$ Teste $\mathrm{t}$ independente $(\Delta \mathrm{CFG}-\Delta \mathrm{MMII}) ; \Delta \%=$ percentual de diferença entre a medida pré e pós treinamento. $\mathrm{D}=$ direito; $\mathrm{E}=$ esquerdo. Fuente: elaboração própria

Em seguida, comparou-se as diferenças dos resultados pós e pré treinamento das medidas e testes de cada programa, por teste $t$ independente. Assim, as variáveis que apresentaram diferença significativa nos resultados médios das avaliações após o treinamento foram as de resistência muscular e flexibilidade, ambas favoráveis ao programa de treinamento MMII.

As participantes do programa CFG apresentaram resultado médio inferior aos da avaliação inicial, nas variáveis de resistência muscular.

Tabela 2 - Comparação da variação dos scores de Qualidade de Vida pré e pós treinamento nos dois programas por Teste $t$ independente, Recife-PE/Brasil, 2020.

\begin{tabular}{|c|c|c|c|c|c|c|c|c|c|c|c|}
\hline \multirow{2}{*}{$\begin{array}{c}\text { Score } \\
\text { VEINES }\end{array}$} & \multicolumn{5}{|c|}{ Programa CFG $(n=9)$} & \multicolumn{5}{|c|}{ Programa MMII $(\mathrm{n}=11)$} & \multirow{2}{*}{$p$} \\
\hline & Pré & DP & Pós & DP & $? \%$ & Pré & DP & Pós & DP & $? \%$ & \\
\hline Sym & 54,21 & 5,00 & 49,10 & 9,08 & $-4 \%$ & 47,18 & 7,91 & 50,05 & 5,67 & $6 \%$ & 0,14 \\
\hline QoL & 51,93 & 4,05 & 50,19 & 4,71 & $-3 \%$ & 48,59 & 5,34 & 49,78 & 2,71 & $2 \%$ & 0,37 \\
\hline
\end{tabular}

$\Delta \%=$ percentual de diferença entre a medida pós e pré treinamento. Fuente: elaboração própria

Nos escores de qualidade de vida, ao comparar a variação relativa antes e após o treinamento, maior percentual de variação tanto em sintomas quanto em QV global nas participantes do programa MMII, 
enquanto as do programa CFG apresentaram variação negativa em comparação à avaliação pré. $\mathrm{Na}$ análise por teste $t$, os resultados não apresentaram diferença significativa (tabela 2 ).

Figura 2 - Resultados individuais das participantes no questionário VEINES-Sym, pré e pós treinamento nos programas CFG $(\mathrm{n}=9)$ e MMII $(\mathrm{n}=11)$, Recife-PE/Brasil, 2020.

Programa CFG

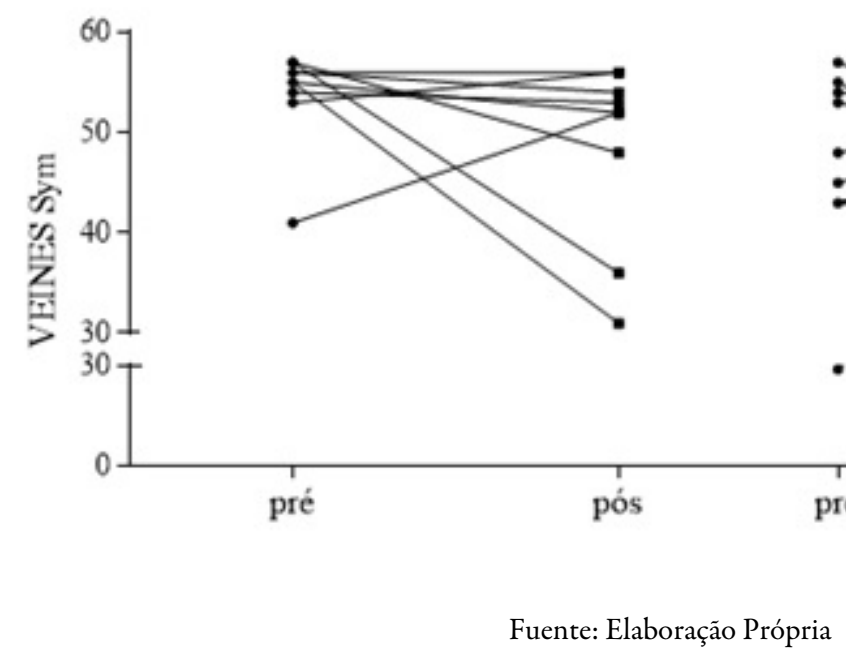

Programa MMII

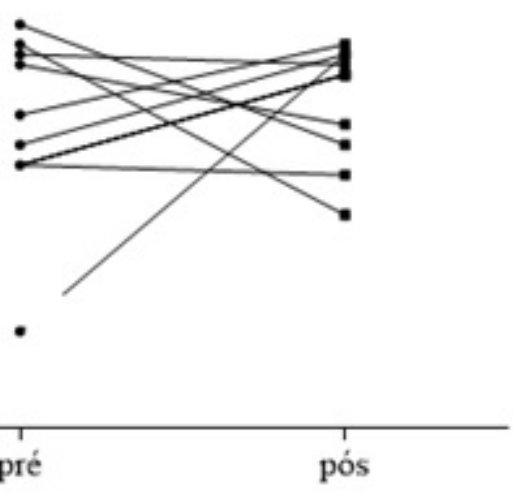

Analisando os dados individuais do questionário VEINES, apresentados na figura 2, encontra-se resultados mais favoráveis ao programa de treinamento MMII, no qual 54,5\% das participantes apresentam scores superiores ao período pré treinamento tanto no domínio de sintomas como em qualidade de vida geral. Dentre as participantes do programa CFG, esse percentual ficou em 44,4\% para o domínio de sintomas e $22,2 \%$ para o de qualidade de vida geral.

\section{Discussão}

As mulheres participantes de programas de exercícios físicos, orientados com foco específico na musculatura dos membros inferiores, beneficiam-se em termos de aptidão física, apresentando melhora nos testes a que foram submetidas. Esse achado, corrobora com outros estudos que utilizam exercícios voltados para musculatura da panturrilha resultando em benefícios quanto a aspectos gerais da doença venosa como atenuação de sintomas e cicatrização de úlceras em pacientes mais graves (C. E. Q. Belczak, Cavalheri, De Godoy, Caffaro, \& Belczak, 2007; Caggiati, De Maeseneer, Cavezzi, Mosti, \& Morrison, 2018).

Após o período de treinamento sob o programa de condicionamento físico geral, além dos resultados inferiores ao programa MMII nas variáveis de aptidão física e qualidade de vida, houve redução nos resultados médios apresentados em relação a avaliação inicial em flexibilidade e resistência muscular. A menor frequência de prática de exercícios de resistência de membros inferiores pode ter sido determinante para esta redução. $\mathrm{Na}$ avaliação de resistência aeróbica, embora o resultado seja positivo, ainda se mostra inferior ao programa MMII.

O programa MMII, apresentou aumento significativo nas variáveis de flexibilidade de tornozelo e resistência muscular da panturrilha. Achados que utilizaram programas de treino, com exercícios de resistência, visando o fortalecimento de membros inferiores, como o estudo de O’Brien, Edwards, Stewart, \& Gibbs (2012), que observaram melhora na função da bomba muscular da panturrilha e amplitude de movimento do tornozelo no grupo que realizou exercícios de elevação de calcanhar durante 12 semanas, em relação ao grupo controle. 
A melhoria significativa em flexibilidade favorável ao programa MMII, tem influência positiva na população com DVC, uma vez que estudos apontam que melhores índices de flexibilidade na articulação do tornozelo estão associados a melhor funcionalidade da musculatura da panturrilha (Davies et al., 2007), ao passo que a redução da flexibilidade desta articulação pode limitar a ação dessa bomba muscular, e agravar a doença resultando em ulcerações de difícil tratamento (Lopes, Figueiredo, Ávila, Soares, \& Dionisio, 2013).

O Teste de Ponta de pé é utilizado para avaliar a função da bomba muscular da panturrilha em pessoas com DVC (Pereira et al., 2015; van Uden, van der Vleuten, Kooloos, Haenen, \& Wollersheim, 2005), neste estudo, apresentou resultado significativo tanto no número de repetições, quanto no tempo de execução, denotando melhoria da resistência dessa musculatura dentre as participantes do programa MMII. No mesmo sentido, uma intervenção de 6 semanas de exercício para fortalecimento da panturrilha, encontrou melhoria na função venosa e muscular da BMP, medida por Pletismografia e dinamometria (Yang, Vandongen, \& Stacey, 1999). A melhora no desempenho no TPP após intervenção com exercício físico também foi encontrada num estudo de caso em que uma mulher submetida a um programa de fortalecimento de panturrilha e condicionamento aeróbico por 15 semanas apresentou incremento aeróbico e na hemodinâmica venosa, inclusive, melhores resultados em qualidade de vida, com ausência de dor após a intervenção (Amorim et al., 2014).

Embora sejam observados melhores resultados entre as participantes do programa MMII nas demais variáveis como IMC, perimetria de panturrilha, resistência aeróbica e trabalho muscular, não houve diferença significativa, ao comparar os resultados dos dois programas.

Em termos de qualidade de vida, ao final da intervenção, não se observou diferença estatística entre os programas de treinamento, ainda que, no grupo do programa MMII, maior percentual de participantes alcançasse resultados individuais superiores ao período pré-treinamento, tanto para o domínio de sintomas, quanto para qualidade de vida geral. Estudo de Ercan et al., (2017), em intervenção de 12 semanas com 27 indivíduos, encontrou melhoria na qualidade de vida e escala de dor. Em outro estudo de caso, uma mulher de 41 anos com classificação C4 da DVC, após 30 sessões de treino envolvendo caminhada e exercícios de alongamento e fortalecimento de MMII, apresentou melhora em qualidade de vida, destacando-se os domínios de dor e habilidades físicas (Lima et al., 2002). Embora, neste estudo, a variação positiva no VEINES não tenha sido significativa, é importante destacar que a melhora em qualquer grau de sintomas de uma doença é relevante para os pacientes que a alcançam individualmente.

Embora a prática de exercícios físicos tenha efeito positivo no controle de sinais e sintomas da DVC, o que pode refletir numa melhor percepção da qualidade de vida, há estudos relatando que 12 semanas possam não ser tempo suficiente para gerar mudanças em termos de qualidade de vida (Ercan et al., 2017; O'Brien, Finlayson, Kerr, \& Edwards, 2017).

No presente estudo é, ainda, importante destacar que, por já serem praticantes de atividades físicas, foi possível observar, já na avaliação inicial, os scores em qualidade de vida das participantes eram positivos em ambos os grupos, permanecendo assim após o período de treinamento, não havendo diferença estatística.

Como pontos fortes deste estudo, entende-se que um ensaio de caráter experimental, aplicado em programa público de atividades físicas com demanda espontânea permite identificar na prática cotidiana os efeitos dos programas de treinamento, ampliando sua validade externa. Por se tratar de uma intervenção em grupos, o resultado positivo encontrado, permite considerar uma ampliação do acesso à atividade física enquanto parte do tratamento da DVC em detrimento a uma intervenção individualizada, consequentemente mais custosa.

Por outro lado, a amostra limitada, dificulta uma análise mais criteriosa quanto a estratificação de grupos por idade, ou severidade da DVC, impedindo um aprofundamento quanto a efetividade dos programas sugeridos. O fato das participantes deste estudo já utilizarem programa de exercício em média a 2 anos, pode ter interferência num melhor nível de aptidão física dessas mulheres, o que por sua vez pode auxiliar 
no controle DVC, uma vez que a prática regular do exercício físico, é apontada em estudos como um fator protetor para evitar a progressão da DVC a estágios mais severos (Alberti et al., 2008).

A avaliação realizada na Universidade, longe de alguns dos polos de intervenção, parece ter contribuído com a dificuldade de retorno das voluntárias para reavaliação, resultando em perda amostral.

Em estudos posteriores, essas analises podem ser aprofundadas, com amostras maiores, período de intervenção mais longo e com grupo controle, permitindo melhor avaliação do efeito do treinamento sobre os participantes, possibilitando ainda estratificar o grupo pelo grau de severidade da DVC e por idade, afim de possibilitar uma atuação prática cada vez mais adequada, segura e eficiente.

\section{Conclusão}

Um programa de exercício físico específico para fortalecimento de membros inferiores mostrou-se capaz de produzir melhores resultados em termos de aptidão física em mulheres com DVC, quando comparado à um programa de condicionamento físico geral, e resultados não significativos quanto a possível melhora na qualidade de vida, sendo necessários novos estudos nesse sentido.

\section{Agradecimentos}

À Secretaria de Saúde do Município do Recife, especialmente aos profissionais do Programa Academia da Cidade que colaboraram na condução da intervenção; Aos instrutores do Projeto Vida Ativa da UFPE; à equipe do Laboratório de Cinesiologia e Avaliação Funcional da UFPE (LACAF).

\section{ReFERÊNCIAS}

Alberti, L. R., Petroianu, A., Corrêa, D., \& Silva, T. F. (2008). Efeito da Actividade Física na Insuficiência Venosa Crônica dos Membros Inferiores. Acta Médica Portuguesa, 21(3), 215-220. Retrieved from http://www.actam edicaportuguesa.com/revista/index.php/amp/article/view/788

Amorim, G., Samora, R., Ramos, M. F., Marchesani, R., Clara, A., Lages, R., ... Pires, D. O. (2014). Treinamento de Resistência da Musculatura da Panturrilha em Um Caso Atípico de Insuficiência Venosa Crônica. Revista de Saúde Pública Do SUS/MG, 2(2).

Belczak, C. E. Q., Cavalheri, G., De Godoy, J. M. P., Caffaro, R. A., \& Belczak, S. Q. (2007). Relação entre a mobilidade da articulação talocrural e a úlcera venosa. Jornal Vascular Brasileiro, 6(2), 149-155. https://doi.org/10.1590/ S1677-54492007000200009

Belczak, C., Godoy, J., Seidel, A., Silva, J., Cavalheri-Junior, G., \& Belczak, S. (2004). Influencia da atividade diária na volumetria de membros inferiores. Jornal Vascular Brasileiro, 3(4), 304-310.

Caggiati, A., De Maeseneer, M., Cavezzi, A., Mosti, G., \& Morrison, N. (2018). Rehabilitation of patients with venous diseases of the lower limbs: State of the art. Phlebology, 10(33), 663-671. https://doi.org/10.1177/02683555 18754463

Davies, J. A., Bull, R. H., Farrelly, I. J., \& Wakelin, M. J. (2007). A home-based exercise programme improves ankle range of motion in long-term venous ulcer patients. Phlebology, 22(2), 86-89. https://doi.org/10.1258/02683 5507780346178

Ercan, S., Çetin, C., Yavuz, T., Demir, H. M., \& Atalay, Y. B. (2017). Effects of isokinetic calf muscle exercise program on muscle strength and venous function in patients with chronic venous insufficiency. Phlebology, 33(4), 261266. https://doi.org/10.1177/0268355517695401

Kan, Y. M., \& Delis, K. T. (2001). Hemodynamic effects of supervised calf muscle exercise in patients with venous leg ulceration: A prospective controlled study. Archives of Surgery, 136(12), 1364-1369. https://doi.org/10.10 01/archsurg.136.12.1364 
Leal, F. de J., Couto, R. C., Silva, T. P. da;, \& Tenório, V. de O. (2015). Vascular physiotherapy in treatment of chronic venous disease. Jornal Vascular Brasileiro, 14(3), 224-230.

Leal, F. de J., Soares, L. M. S., Couto, R. C., Moraes, S. G. P., da Silva, T. S., \& dos Santos, W. R. (2016). Tratamento fisioterapêutico vascular para a doença venosa crônica: Artigo de revisão. Jornal Vascular Brasileiro, 15(1), $34-$ 43. https://doi.org/10.1590/1677-5449.003215

Lima, R. C. M., Santiago, L., Moura, R. M. F. de, Filaretti, F. A. S., Souza, C. S. A. de, Evangelista, S. S. M., \& Britto, R. R. (2002). Efeitos do fortalecimento muscular da panturrilha na hemodinâmica venosa e na qualidade de vida em um portador de insuficiência venosa crônica. Jornal Vascular Brasileiro, 1(3), 219-226. Retrieved from htt p://www.jvascbr.com.br/02-01-03/02-01-03-219/art04.pdf

Lopes, C. R., Figueiredo, M., Ávila, A. M., Soares, L. M. B. M., \& Dionisio, V. C. (2013). Evaluation of limitations of venous ulcers in legs. Jornal Vascular Brasileiro, 12(1), 5-9. https://doi.org/10.1590/S1677-54492013000 100003

Marrara, K. T., Marino, D. M., \& Jamami, M. (2012). Responsividade do teste do degrau de seis minutos a um programa de treinamento físico em pacientes com DPOC*.J. Bras. Pneumol., 38(5), 579-587.

Monteiro, D. P., Britto, R. R., Fregonezi, G. A. de F., Dias, F. A. L., Silva, M. G. da, \& Pereira, D. A. G. (2017). Reference values for the bilateral heel-rise test. Brazilian Journal of Physical Therapy, 21(5), 344-349. https://doi.org/10 $.1016 /$ j.bjpt.2017.06.002

Moura, R. M. F. de;, Gonçalves, G. S., Navarro, T. P., Britto, R. R., \& Dias, R. C. (2011). Adaptação transcultural do questionário VEINES / QOL-SYM\#: avaliação da qualidade de vida e sintomas na doença venosa crônica. Jornal Vascular Brasileiro, 10(1), 17-23.

Moura, R. M. F., Gonçalves, G. S., Navarro, T. P., Britto, R. R., \& Dias, R. C. (2008). Correlação entre classificação clínica ceap e qualidade de vida na doença venosa crônica. Revista Brasileira de Fisioterapia, 14(2), 1-7.

Murayama, R., Carraro, L. D., Galvanin, T., Izukawa, N. M., Umeda, I., \& Oliveira, M. F. (2014). Insuficiência vascular periférica compromete a capacidade funcional no paciente com insuficiência cardíaca. Jornal Vascular Brasileiro, 13(2), 101-107. https://doi.org/10.1590/jvb.2014.053

O’Brien, J., Edwards, H., Stewart, I., \& Gibbs, H. (2012). A home-based progressive resistance exercise programme for patients with venous leg ulcers: A feasibility study. International Wound Journal, 10(4), 389-396. https:// doi.org/10.1111/j.1742-481X.2012.00995.x

O'Brien, J., Finlayson, K., Kerr, G., \& Edwards, H. (2017). Evaluating the effectiveness of a self-management exercise intervention on wound healing, functional ability and health-related quality of life outcomes in adults with venous leg ulcers: a randomised controlled trial. International Wound Journal, 14(1), 130-137. https://doi.or g/10.1111/iwj.12571

Pereira, D. A. G., Lages, A. C. R., Basílio, M. L., Pires, M. C. de O., Monteiro, D. P., \& Navarro, T. P. (2015). Does the heel-rise test explain functional capacity in venous insufficiency? Fisioterapia Em Movimento, 28(1), 61-67. https://doi.org/10.1590/0103-5150.028.001.AO06

Pereira, D. A. G., Oliveira, K. L. de, Cruz, J. O., Souza, C. G. de, \& Cunha Filho, I. T. (2008). Avaliação da reprodutibilidade de testes funcionais na doença arterial periférica. Fisioterapia e Pesquisa, 15(3), 228-234. htt ps://doi.org/10.1590/s1809-29502008000300003

Rabe, E., \& Pannier, F. (2012). Clinical, aetiological, anatomical and pathological classification (CEAP): Gold standard and limits. Phlebology, 27(SUPPL. 1), 114-118. https://doi.org/10.1258/phleb.2012.012S19

Szewczyk, M. T., Jawien, A., Cwajda-Bialasik, J., Cierzniakowska, K., Moscicka, P., \& Hancke, E. (2010). Randomized study assessing the influence of supervised exercises on ankle joint mobility in patients with venous leg ulcerations. Archives of Medical Science, 6(6), 956-963. https://doi.org/10.5114/aoms.2010.19308

van Uden, C. J., van der Vleuten, C. J., Kooloos, J. G., Haenen, J. H., \& Wollersheim, H. (2005). Gait and calf muscle endurance in patients with chronic venous insufficiency. Clinical Rehabilitation, 19(3), 339-344. https://doi. org/10.1191/0269215505cr809oa 
Yang, D., Vandongen, Y. K., \& Stacey, M. C. (1999). Effect of exercise on calf muscle pump function in patients with chronic venous disease. British Journal of Surgery, 86(3), 338-341. https://doi.org/10.1046/j.1365-2168.1999 .00993.x

Youn, Y. J., \& Lee, J. (2019). Chronic Venous Insufficiency and Varicose Veins. The Korean Journal of Internal Medicine, 34(2), 269-283. https://doi.org/10.1056/NEJMcp0802444 